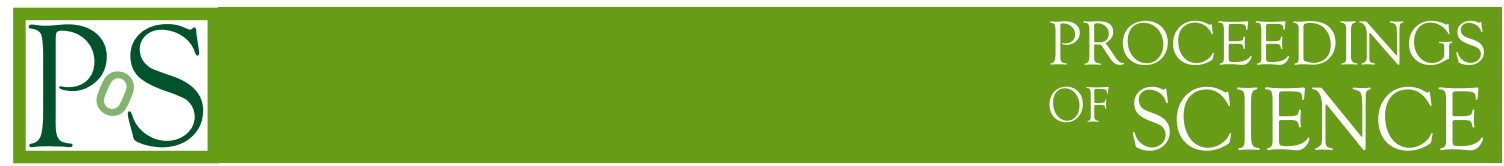

\title{
Decays of mesons with charm quarks on the lattice
}

\author{
A. Ali Khan ${ }^{* a \dagger}$, V. Braun ${ }^{a}$, T. Burch ${ }^{a}$, M. Göckeler ${ }^{a}$, G. Lacagnina ${ }^{b}$, A. Schäfer ${ }^{a}$, and \\ G. Schierholz ${ }^{c}$ \\ ${ }^{a}$ Institut für Theoretische Physik, Universität Regensburg, 93040 Regensburg, Germany \\ ${ }^{b}$ Dipartimento di Fisica, Università di Pisa and INFN, Pisa, Italy \\ ${ }^{c}$ John von Neumann-Institut für Computing NIC, Deutsches Elektronen-Synchrotron DESY, \\ 15738 Zeuthen, Germany \\ Deutsches Elektronen-Synchrotron DESY, 22603 Hamburg, Germany \\ E-mail: arifa.ali-khanephysik.uni-regensburg.de
}

\begin{abstract}
We investigate mesons containing charm quarks on fine lattices with $a^{-1} \sim 5 \mathrm{GeV}$. The quenched approximation is employed using the Wilson gauge action at $\beta=6.6$ and nonperturbatively $O(a)$ improved Wilson quarks. We present results for decay constants using various interpolating fields and give preliminary results for form factors of semileptonic decays of $D_{s}$ mesons to light pseudoscalar mesons.
\end{abstract}

The XXV International Symposium on Lattice Field Theory

July 30-4 August 2007

Regensburg, Germany

\footnotetext{
${ }^{*}$ Speaker.

${ }^{\dagger}$ Address after 1/10/2007: Department of Physics, Faculty of Science, Taiz University, Taiz, Yemen Republic.
} 


\section{Introduction}

There are current experimental and theoretical activities to investigate the decays of heavylight hadrons. Study of their weak decays is of interest for determining the CKM matrix of quark mixing, and there are theoretical and experimental efforts to test and overconstrain the Standard Model and find signatures of New Physics.

It is of interest to study charmed hadrons on fine lattices where discretization effects are very small. While unquenched data from very fine lattices is not yet easy to obtain, the QCDSF collaboration has undertaken a quenched calculation of charmed and also bottom decay constants, weak matrix elements and meson spectra on a quenched lattice with an inverse lattice spacing of $a^{-1} \simeq 5 \mathrm{GeV}$. Results on heavy-light and light meson decay constants have been presented in a previous paper [1]. A particularly interesting result of this calculation was a relatively low value of the decay constant $f_{D_{s}}$. We have analyzed further matrix elements and would like to present our findings here. In Section 2 we give a short description of simulation and analysis details. Our calculation of the pseudoscalar decay constants using two different interpolating operators for the pseudoscalar meson is discussed in Section 3. In Section 4 we give preliminary results for form factors of semileptonic decays of pseudoscalar heavy-light mesons to pseudoscalar light mesons.

\section{Simulation parameters}

Our quenched lattices are generated using the Wilson gauge field action at $\beta=6.6$. The lattice spacing, determined using the Sommer parameter $r_{0}=0.5 \mathrm{fm}$ from [2], is $a=0.040 \mathrm{fm}$ or $a^{-1}=4.97 \mathrm{GeV}$. The lattice size is $40^{3} \times 80$. For the results reported here, we have analyzed 114 gauge field configurations.

We use $O(a)$ improved Wilson quarks. The value of the clover coefficient has been determined nonperturbatively by Ref. [3]. We work with three 'light' and four 'heavy' hopping parameters. Their values and the corresponding quark masses can be found in Table 1.

\begin{tabular}{lccccccc}
\hline$\kappa$ & 0.13519 & 0.13498 & 0.13472 & 0.13000 & 0.12900 & 0.12100 & 0.11500 \\
$a \tilde{m}_{q}$ & 0.0076 & 0.013 & 0.020 & 0.14 & 0.16 & 0.31 & 0.37 \\
$M[\mathrm{GeV}]$ & 0.55 & 0.69 & 0.87 & 2.8 & 3.2 & 5.6 & 7.2 \\
\hline
\end{tabular}

Table 1: Hopping parameters, the corresponding $O(a)$ improved quark masses (see Eq. (2.1)) and approximate pseudoscalar meson masses (denoted as $M$ ).

In our analysis we parameterize the light quark masses using the $O(a)$ improved quark mass

$$
a \tilde{m}_{q}=\left(1+b_{m} a m_{q}\right),
$$

where $a m_{q}=\frac{1}{2}\left(\frac{1}{\kappa}-\frac{1}{\kappa_{c r i t}}\right)$. The nonperturbatively determinated value of $b_{m}$ is taken from Ref. [4]. As a measure for the heavy quark masses we use the mass of the heavy-light meson, where the light quark mass is extrapolated to the average of the $u$ and $d$ quark mass.

We calculate matrix elements of the $O(a)$ improved axial vector current containing the quarks $q_{1}$ and $q_{2}$ :

$$
A_{4}^{I}=Z_{A}\left(1+b_{A} a m_{q}\right)\left(A_{4}+a c_{A} \partial_{4} P\right),
$$


where $A_{4}(x)=\bar{q}_{1}(x) \gamma_{4} \gamma_{5} q_{2}(x)$ is the local axial vector lattice current, and $P(x)=\bar{q}_{1}(x) \gamma_{5} q_{2}(x)$ the local pseudoscalar density. With $\partial_{4}$ we denote the symmetric lattice derivative. We use the nonperturbatively determined values for $c_{A}$ from [3] and for $Z_{A}$ from [5]. For the coefficient $b_{A}$ of the bare quark mass $a m_{q}$, we use the value of Ref. [6], calculated in one-loop perturbation theory, and the boosted lattice coupling.

The $O(a)$ improved vector current is given by

$$
V_{\mu}^{I}=Z_{V}\left(1+a m_{q} b_{V}\right)\left(V_{\mu}+i a c_{V} \partial_{v} T_{\mu v}\right)
$$

where $V_{\mu}=\bar{q}_{1} \gamma_{\mu} q_{2}$ and $T_{\mu \nu}=\bar{q}_{1} \frac{i}{2}\left[\gamma_{\mu}, \gamma_{v}\right] q_{2}$. We use for $Z_{V}$ and $b_{V}$ the nonperturbatively calculated results from [7]. For the correction due to the tensor current we have only made a preliminary order-of-magnitude estimate, using a rational interpolation [8] of the nonperturbative values for $c_{V}$ from [9].

\section{Pseudoscalar decay constants}

We calculate the decay constant $f$ of pseudoscalar mesons $M$ at zero momentum from

$$
f=\frac{1}{M}\left\langle 0\left|A_{4}^{I}\right| M\right\rangle \text {. }
$$

Previously ([1]) we used operators of the type $P$ to project at the source onto the pseudoscalar meson. Since different correlation functions may be subject to different systematic errors, we

\begin{tabular}{|c|c|c|c|c|c|}
\hline & & \multicolumn{2}{|c|}{$a m_{P S}$} & \multicolumn{2}{|c|}{$a f^{(0)}$} \\
$\kappa_{1}$ & $\kappa_{2}$ & $A 4 P$ & $A 4 A 4$ & $A 4 P$ & $A 4 A 4$ \\
\hline 0.11500 & 0.13519 & $0.8363(15)$ & $0.8350(14)$ & $0.0371(11)$ & $0.0367(15)$ \\
0.12100 & 0.13519 & $0.6676(13)$ & $0.6666(13)$ & $0.0417(14)$ & $0.0404(15)$ \\
0.12900 & 0.13519 & $0.4065(11)$ & $0.4058(12)$ & $0.0475(13)$ & $0.0465(14)$ \\
0.13000 & 0.13519 & $0.3685(12)$ & $0.3678(12)$ & $0.0478(13)$ & $0.0469(13)$ \\
0.11500 & 0.13498 & $0.8431(12)$ & $0.8415(12)$ & $0.0383(12)$ & $0.0377(14)$ \\
0.12100 & 0.13498 & $0.6747(11)$ & $0.6735(11)$ & $0.0429(12)$ & $0.0418(14)$ \\
0.12900 & 0.13498 & $0.4145(10)$ & $0.4137(09)$ & $0.0488(15)$ & $0.0480(14)$ \\
0.13000 & 0.13498 & $0.3765(09)$ & $0.3756(09)$ & $0.0490(13)$ & $0.0482(13)$ \\
0.11500 & 0.13472 & $0.8517(11)$ & $0.8503(11)$ & $0.0402(12)$ & $0.0393(14)$ \\
0.12100 & 0.13472 & $0.6836(10)$ & $0.6826(10)$ & $0.0446(13)$ & $0.0437(15)$ \\
0.12900 & 0.13472 & $0.4242(08)$ & $0.4234(08)$ & $0.0508(13)$ & $0.0497(14)$ \\
0.13000 & 0.13472 & $0.3866(08)$ & $0.3860(08)$ & $0.0507(13)$ & $0.0501(14)$ \\
\hline
\end{tabular}

Table 2: Pseudoscalar heavy-light meson masses and decay constants at $O\left(a^{0}\right)$ from $\left\langle A_{4} P\right\rangle$ and $\left\langle A_{4} A_{4}\right\rangle$ correlation functions at the simulated hopping parameters.

would like to determine the matrix elements of $A_{4}$ also using the temporal component of the axial vector current operator, i.e. from correlation functions of the form

$$
C_{A 4 A 4}^{S i}(t)=\sum_{\vec{x}}\left\langle A_{4}^{i}(\vec{x}, t) A_{4}^{S \dagger}(0)\right\rangle .
$$


The index $i$ stands for local $(i=L)$ or Jacobi smeared $(i=S)$ operators. If we write the decay constant as

$$
f=Z_{A}\left(1+b_{A} a m_{q}\right)\left(f^{(0)}+a c_{A} f^{(1)}\right),
$$

we find the unimproved contribution $f^{(0)}$ from the correlation function in Eq. (3.2).

We extract masses and amplitudes from single state fits to the correlation functions. The pseudoscalar meson masses and the results for $f^{(0)}$ are compared for heavy-light mesons at the simulated quark masses in Table 2 with the corresponding values from [1]. The results agree within the statistical errors.

\begin{tabular}{cc|cc}
\hline \multicolumn{2}{c}{ From A4PS correlators (Ref.[1]) } & \multicolumn{2}{c}{ From $A 4 A 4$ correlators (this work) } \\
\hline \multicolumn{4}{c}{ Light meson decay constants } \\
$f_{\pi}[\mathrm{MeV}]$ & $f_{K}[\mathrm{MeV}]$ & $f_{\pi}[\mathrm{MeV}]$ & $f_{K}[\mathrm{MeV}]$ \\
$140(4)$ & $152(4)$ & $138(6)$ & $150(5)$ \\
\hline \multicolumn{4}{c}{ Heavy-strange meson decay constants } \\
$f_{D_{s}}[\mathrm{MeV}]$ & $f_{B_{s}}[\mathrm{MeV}]$ & $f_{D_{s}}[\mathrm{MeV}]$ & $f_{B_{s}}[\mathrm{MeV}]$ \\
$220(6)(5)(11)$ & $205(7)(26)(17)$ & $217(5)$ & $204(9)$ \\
\hline \multicolumn{4}{c}{ Heavy-light meson decay constants } \\
$f_{D}[\mathrm{MeV}]$ & $f_{B}[\mathrm{MeV}]$ & $f_{D}[\mathrm{MeV}]$ & $f_{B}[\mathrm{MeV}]$ \\
$206(6)(3)(22)$ & $190(8)(23)(25)$ & $202(6)$ & $191(9)$ \\
\hline
\end{tabular}

Table 3: Results on decay constants from different correlators. The first error is statistical, the second error on the results from [1] is systematic and the third from the uncertainty in the experimental value of $r_{0}$. We estimate the systematic errors in the new results to be similar.

We fit the matrix elements to linear functions in the light quark masses and, where applicable, to quadratic functions in the heavy quark masses, using the method described in [1]. To determine the physical light, strange and heavy quark masses we also use the same method as for the central values given in [1]. The decay constant results are listed in Table 3. We take over the errors for the heavy-light decay constants from [1], including uncertainties from setting the quark masses to the physical values, discretization effects, errors in the nonperturbative renormalization constants and from finite volume effects. A $10 \%$ uncertainty in the experimental value of $r_{0}$ is also included. We did not yet perform the same reanalyses for the data from the $A_{4} A_{4}$ correlators and just assume that the systematic errors are very similar. We did not attempt an estimate of the systematic error for the light meson decay constants.

Our result for $f_{D_{s}}$ is still smaller than the recently updated experimental value of the $D_{s}$ decay constant of 275(10) stat $(5)_{\text {syst }} \mathrm{MeV}$ by CLEO [10].

\section{Semileptonic form factors}

We describe preliminary results for matrix elements of semileptonic decay of pseudoscalar

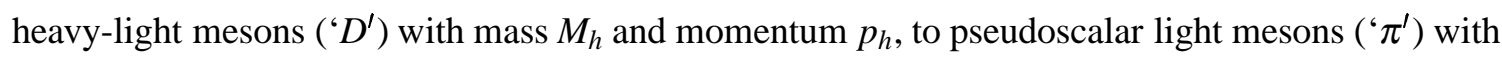
mass $M_{l}$ and momentum $p_{l}$. The decay goes via a vector current $V_{\mu}(x)$ with the matrix element

$$
\mathscr{M}_{\mu}=\left\langle\pi\left(p_{l}\right)\left|V_{\mu}(0)\right| D\left(p_{h}\right)\right\rangle .
$$


We would like to determine the two form factors which are often used to parameterize the matrix element

$$
\mathscr{M}_{\mu}=\left(p_{h}+p_{l}-\Delta q\right)_{\mu} F_{+}\left(q^{2}\right)+\Delta q_{\mu} F_{0}\left(q^{2}\right),
$$

with $q=p_{h}-p_{l}$ and $\Delta=\left(M_{h}^{2}-M_{l}^{2}\right) / q^{2}$ [13]. The matrix element can be extracted from the three
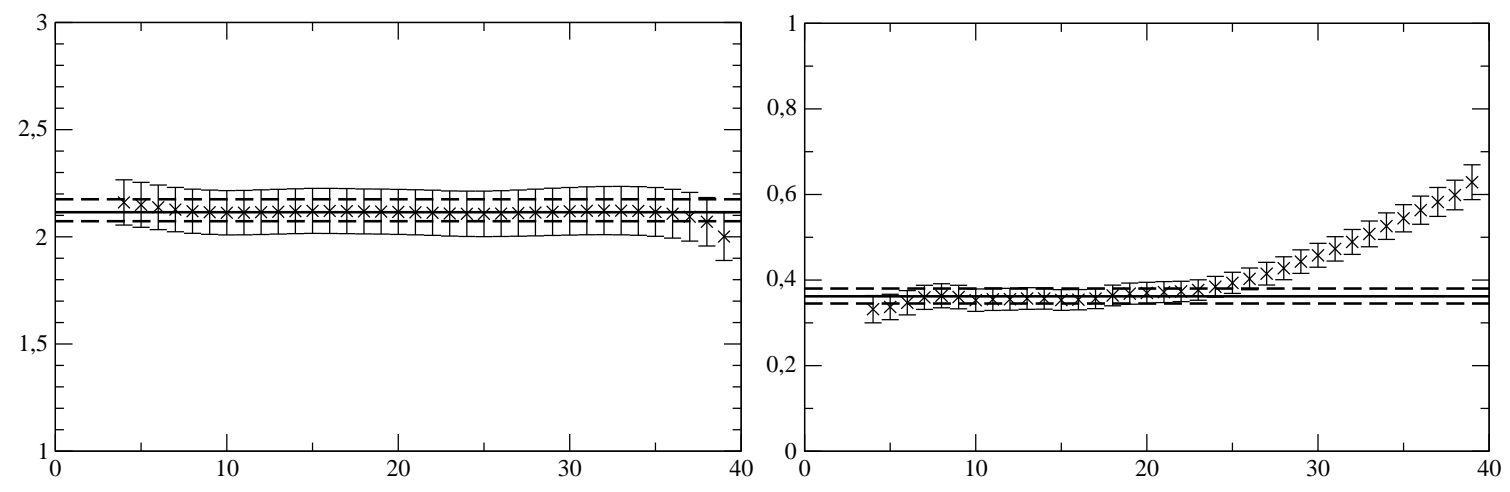

Figure 1: 'Divided' three point functions at the hopping parameter of the heavy quark $\kappa_{h}=0.12900$ and the hopping parameter $\kappa=0.13498$ for the light and spectator quark. The meson momenta are $\vec{p}_{h}=(1,0,0)$ and $\vec{q}=(1,0,0)$ in units of $2 \pi / L$. The crosses denote the data points, the solid lines the central values and the dashed lines the error bars of the fit. On the left we show $\left|\mathscr{M}_{4}\right|$ and on the right $\left|\mathscr{M}_{1}\right|$.

point correlation function of a pseudoscalar light meson at time zero, a vector current at time $t_{x}$ and a pseudoscalar heavy-light meson at time $t_{y}=T / 2$, where $T$ is the time extent of the lattice:

$$
C_{\mu}^{(3)}\left(t_{x}, t_{y}\right)=\sum_{\vec{x}, \vec{y}} e^{-i \vec{p} h \cdot \vec{y}} e^{i \vec{q} \cdot \vec{x}}\left\langle P_{h}^{S}\left(\vec{y}, t_{y}\right) V_{\mu}\left(\vec{x}, t_{x}\right) P_{l}^{S}(0)\right\rangle .
$$

$P_{h}^{S}$ and $P_{l}^{S}$ are Jacobi smeared operators with the structure $\bar{q} \gamma_{5} q_{l}$ and $\bar{q}_{h} \gamma_{5} q$. With $q_{h}$ we denote the heavy quark, with $q_{l}$ the light quark, and with $q$ the spectator quark. The vector current is of the type $V_{\mu}=\bar{q}_{l} \gamma_{\mu} q_{h}$.

The large time behavior $\left(0 \ll t_{x} \ll t_{y}\right)$ or $\left(0 \ll t_{y} \ll t_{x}\right)$ of the three point function is given by

$$
\begin{aligned}
& C_{\mu}^{(3)}\left(t_{x}, t_{y}\right) \longrightarrow \frac{Z_{l}^{S}}{2 E_{l}} \frac{Z_{h}^{S}}{2 E_{h}} e^{-E_{l} t_{x}} e^{-E_{h}\left(t_{y}-t_{x}\right)}\left\langle\pi\left(p_{l}\right)\left|V_{\mu}\right| D\left(p_{h}\right)\right\rangle, t_{x}<t_{y}, \\
& C_{\mu}^{(3)}\left(t_{x}, t_{y}\right) \longrightarrow \pm \frac{Z_{h}^{S}}{2 E_{h}} \frac{Z_{l}^{S}}{2 E_{l}} e^{-E_{l}\left(T-t_{x}\right)} e^{-E_{h}\left(t_{x}-t_{y}\right)}\left\langle\pi\left(p_{l}\right)\left|V_{\mu}\right| D\left(p_{h}\right)\right\rangle, t_{x}>t_{y},
\end{aligned}
$$

where the prefactors are given by $Z_{h}^{S}=\left|\left\langle 0\left|P_{h}^{S}\right| D\left(\vec{p}_{h}\right)\right\rangle\right|$ and $Z_{l}^{S}=\left|\left\langle 0\left|P_{l}^{S}\right| \pi\left(\vec{p}_{l}\right)\right\rangle\right|$. The correlation functions are symmetric or antisymmetric around $T / 2$. We bin over the contributions from $t_{x}>t_{y}$ and $t_{x}<t_{y}$, and divide the three point functions by the prefactors extracted from fits to smearedsmeared two point functions in a bootstrap loop. The 'divided' three point function is fitted to a constant to extract the matrix element. An example is shown in Fig. 1. In this preliminary analysis we considered in particular momentum combinations where the $D$ and the $\pi$ both have spatial momenta $\leq 2 \pi / L$, where $L$ is the spatial lattice extent, and the momenta are aligned, i.e.:

$$
\begin{aligned}
& \vec{p}_{h}=(0,0,0),-\vec{q}=(0,0,0),(1,0,0),(0,1,0),(0,0,1),(-1,0,0), \\
& \vec{p}_{h}=(1,0,0),-\vec{q}=(0,0,0),(-1,0,0),(-2,0,0),
\end{aligned}
$$


in units of $2 \pi / L$. The kinematic conditions are such that for each heavy and light meson mass, each different combination of $\vec{p}_{h}$ and $\vec{q}$ leads to a different value of $q^{2}=\left(E_{h}-E_{l}\right)^{2}-\vec{q}^{2}$. To extract the form factors for each given value of $q^{2}$ separately, without making a guess for the $q^{2}$ dependence of the form factors, we only have the four Lorentz components of the matrix elements $\mathscr{M}_{\mu}$ at our disposition.

We have exploited the fact that the form factors appear in the spatial and temporal components of $\mathscr{M}_{\mu}$ with different momentum prefactors, so we obtain a linear equation system which we can solve for $F_{0}$ and $F_{+}$.

Results for the form factors at several heavy $\kappa$ values and with the light and spectator quark mass close to the strange quark mass are shown in Fig. 2. For a preliminary estimate of the statistical error we use error propagation of the bootstrap errors of the spatial and temporal components of $\mathscr{M}_{\mu}$. In the results shown in the Figure, the $O(a)$ correction to the vector current (see Eq. (2.3)) is not yet included. We have however estimated its magnitude to be at most a few percent for heavy quark masses around the charm. Although the quark masses are not tuned to the physical values matching $K$ mesons, and the results correspond to decays of $D_{s}$ to pseudoscalar strange mesons, we attempt to compare them to experiment and other lattice results. For $\kappa=0.12900$, close to $\kappa_{\text {charm }}$, we find $F_{0}(0) \approx F_{+}(0) \approx 0.75$. For $D \rightarrow K$ decays, a recent experimental determination from BABAR has found a value of 0.727(11) [11], a lattice calculation with three flavors of dynamical quarks on a coarser lattice using Fermilab heavy quarks quotes $F_{+}(0)=0.73(8)$ [12], and a quenched calculation on a coarser lattice quotes $F_{+}(0)=0.66(4)$ [13]. So there seems to be at least a rough agreement, but for a more precise result the tuning of quark masses to the physical values will have to be performed.

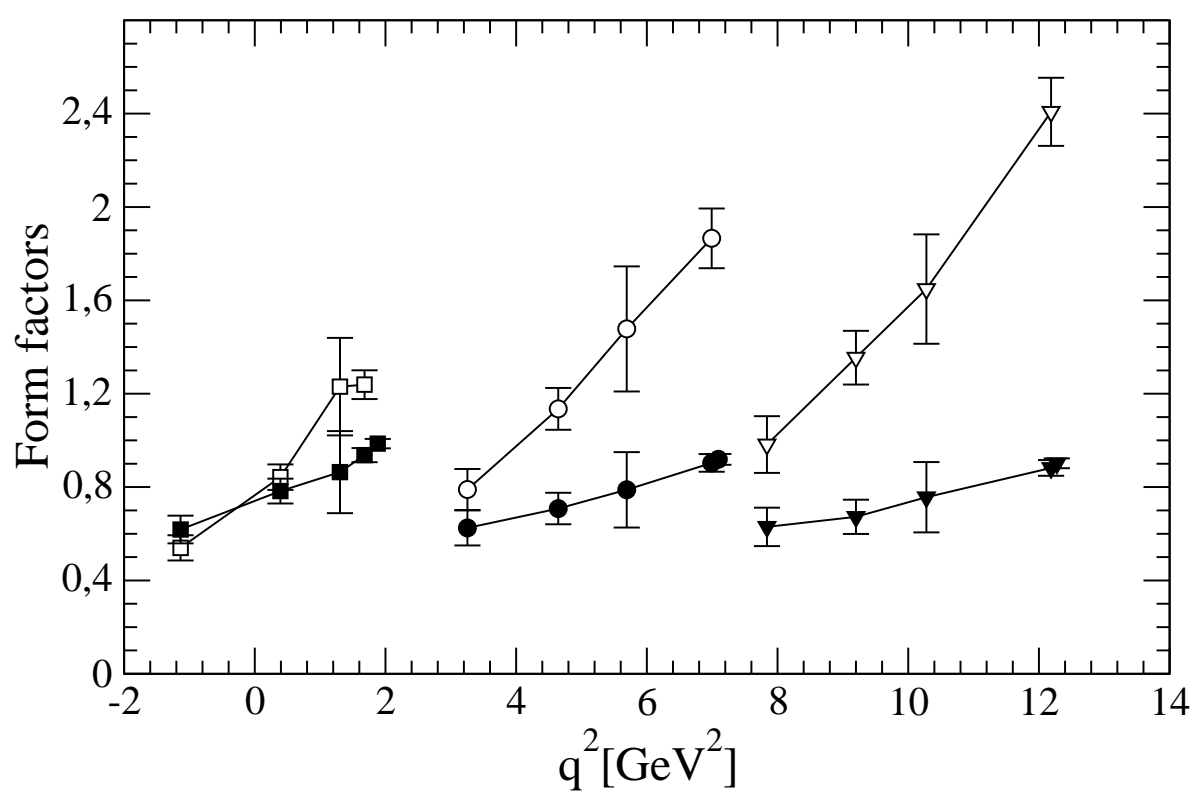

Figure 2: Form factors for semileptonic decays for various heavy quark masses. The light and spectator quark hopping parameters are fixed to the $\kappa$ value 0.13498 . Filled symbols denote $F_{0}$, open symbols $F_{+}$. Squares correspond to $\kappa_{h}=0.129\left(M_{h}=2.1 \mathrm{GeV}\right)$, circles to $\kappa_{h}=0.121\left(M_{h}=3.4 \mathrm{GeV}\right)$, and triangles to $\kappa_{h}=0.115\left(M_{h}=4.2 \mathrm{GeV}\right)$. 


\section{Acknowledgements}

The numerical calculations have been performed on the Hitachi SR8000 at LRZ Munich. This work was supported by DFG (Forschergruppe Gitter-Hadronen-Phänomenologie) and GSI. A.A. thanks the DFG and "Berliner Programm zur Förderung der Chancengleichheit für Frauen in Forschung und Lehre" for financial support.

\section{References}

[1] A. Ali Khan, V. Braun, T. Burch, M. Göckeler, G. Lacagnina, A. Schäfer and G. Schierholz, Phys. Lett. B 652, 150 (2007) [arXiv:hep-lat/0701015].

[2] S. Necco and R. Sommer, Nucl. Phys. B 622, 328 (2002) [arXiv:hep-lat/0108008].

[3] M. Lüscher, S. Sint, R. Sommer, P. Weisz and U. Wolff, Nucl. Phys. B 491, 323 (1997) [arXiv:hep-lat/9609035].

[4] M. Guagnelli, R. Petronzio, J. Rolf, S. Sint, R. Sommer and U. Wolff [ALPHA Collaboration], Nucl. Phys. B 595, 44 (2001) [arXiv:hep-lat/0009021].

[5] M. Lüscher, S. Sint, R. Sommer and H. Wittig, Nucl. Phys. B 491, 344 (1997) [arXiv:hep-lat/9611015].

[6] S. Sint and P. Weisz, Nucl. Phys. B 502, 251 (1997) [arXiv:hep-lat/9704001].

[7] T. Bakeyev, M. Göckeler, R. Horsley, D. Pleiter, P. E. L. Rakow, G. Schierholz and H. Stüben [QCDSF-UKQCD Collaboration], Phys. Lett. B 580, 197 (2004) [arXiv:hep-lat/0305014].

[8] D. Pleiter, Ph.D. thesis.

[9] M. Guagnelli and R. Sommer, Nucl. Phys. Proc. Suppl. 63, 886 (1998) [arXiv:hep-lat/9709088].

[10] S. Stone, arXiv:0709.2706 [hep-ex].

[11] B. Aubert et al. [BABAR Collaboration], arXiv:0704.0020 [hep-ex].

[12] C. Aubin et al. [Fermilab Lattice Collaboration], Phys. Rev. Lett. 94, 011601 (2005) [arXiv:hep-ph/0408306].

[13] A. Abada, D. Becirevic, P. Boucaud, J. P. Leroy, V. Lubicz and F. Mescia, Nucl. Phys. B 619, 565 (2001) [arXiv:hep-lat/0011065]. 